\title{
Impact of Adding Telephone-Based Care Coordination to Standard Telephone-Based Smoking Cessation Counseling Post-hospital Discharge: a Randomized Controlled Trial
}

\author{
Edward F. Ellerbeck, MD, MPH ${ }^{\top}\left(\mathbb{D}\right.$, Lisa Sanderson Cox, $P h D^{7}$, \\ Siu-kuen Azor Hui, PhD, MSPH², John Keighley, PhD³, Tresza D. Hutcheson, PhD', \\ Sharon A. Fitzgerald, $\mathrm{MPH}^{7}$, A. Paula Cupertino, $P h D^{7}, \mathrm{~K}$. Allen Greiner, $\mathrm{MD}, \mathrm{MPH}{ }^{4}$, \\ Nancy A. Rigotti, MD ${ }^{5}$, Nancy Houston Miller, $R N, B S N^{6}$, Vance Rabius, $P h D^{7}$, and \\ Kimber $P$. Richter, $P h D, M P H^{7}$
}

\begin{abstract}
'Department of Population Health, University of Kansas Medical Center, Kansas City, KS, USA; ${ }^{2}$ Philadelphia Department of Public Health, Philadelphia, PA, USA; ${ }^{3}$ Department of Biostatistics, University of Kansas Medical Center, Kansas City, KS, USA; ${ }^{4}$ Department of Family Medicine, University of Kansas Medical Center, Kansas City, KS, USA; ${ }^{5}$ Division of General Internal Medicine, Massachusetts General Hospital/Harvard Medical School, Boston, MA, USA; ${ }^{6}$ The LifeCare Company, Menlo Park, CA, USA; ' Department of Behavioral Science, University of Texas MD Anderson

Cancer Center, Houston, TX, USA.
\end{abstract}

BACKGROUND: Cessation counseling and pharmacotherapy are recommended for hospitalized smokers, but better coordination between cessation counselors and providers might improve utilization of pharmacotherapy and enhance smoking cessation.

OBJECTIVE: To compare smoking cessation counseling combined with care coordination post-hospitalization to counseling alone on uptake of pharmacotherapy and smoking cessation.

DESIGN: Unblinded, randomized clinical trial

PARTICIPANTS: Hospitalized smokers referred from primarily rural hospitals

INTERVENTIONS: Counseling only (C) consisted of telephone counseling provided during the hospitalization and post-discharge. Counseling with care coordination (CCC) provided similar counseling supplemented by feedback to the smoker's health care team and help for the smoker in obtaining pharmacotherapy. At 6 months post-hospitalization, persistent smokers were re-engaged with either CCC or C.

MAIN MEASURES: Utilization of pharmacotherapy and smoking cessation at 3, 6, and 12 months post-discharge. KEY RESULTS: Among 606 smokers randomized, 429 (70.8\%) completed the 12-month assessment and 580 $(95.7 \%)$ were included in the primary analysis. Use of any cessation pharmacotherapy between 0 and 6 months (55.2\%) and between 6 and 12 months (47.1\%) postdischarge was similar across treatment arms though use of prescription-only pharmacotherapy between months 6-12 was significantly higher in the CCC group (30.1\%) compared with the C group (18.6\%) (RR, 1.61 (95\% CI, $1.08,2.41)$ ). Self-reported abstinence rates of $26.2 \%$,

Vance Rabius is a retired doctor who worked at the Department of Behavioral Science, University of Texas MD Anderson Cancer Center, Houston, TX, USA

Received April 15, 2019

Accepted July 12, 2019

Published online July 31, 2019
$20.3 \%$, and $23.4 \%$ at months 3,6 , and 12 , respectively, were comparable across the two treatment arms. Of those smoking at month $6,12.5 \%$ reported abstinence at month 12. Validated smoking cessation at 12 months was $19.3 \%$ versus $16.9 \%$ in the CCC and C groups, respectively (RR, 1.13 (95\% CI, 0.80, 1.61)).

CONCLUSION: Supplemental care coordination, provided by counselors outside of the health care team, failed to improve smoking cessation beyond that achieved by cessation counseling alone. Re-engagement of smokers 6 months post-discharge can lead to new quitters, at which time care coordination might facilitate use of prescription medications.

\section{TRIAL REGISTRATION: NCT01063972}

KEY WORDS: smoking cessation; care coordination; transition of care.

J Gen Intern Med 34(12):2804-11

DOI: $10.1007 / \mathrm{s} 11606-019-05220-2$

(c) Society of General Internal Medicine 2019

\section{BACKGROUND}

An estimated 6.5 million smokers are hospitalized each year in the USA. ${ }^{1}$ These hospitalizations provide an opportunity to help smokers quit. ${ }^{2,3}$ Smoking cessation interventions initiated during the hospitalization and continued after discharge can be effective. ${ }^{3,4}$ Smoking cessation is included in hospital quality measures, ${ }^{5}$ but the cessation services provided for hospitalized patients may fall short of the standards that have been shown to be effective.

While smokers may initiate cessation treatment during their hospitalization, care plans initiated during the hospitalization often fail to extend into the outpatient setting. ${ }^{6}$ Transitions of care may be particularly problematic for smoking cessation where there is often confusion about insurance coverage for 
treatment ${ }^{7-9}$ and over-the-counter cessation treatment may not be included among a patient's discharge prescriptions.

Existing telephone-based counseling services generally counsel smokers on the benefits of cessation pharmacotherapy but do not coordinate pharmacotherapy treatment with the smoker's health care provider. ${ }^{10}$ Without help, cost can be a major barrier to obtaining cessation treatment. ${ }^{11,12}$ Without a prescription from a health care provider, smokers cannot take advantage of insurance coverage for smoking cessation medications and their access to free or low cost treatment is often limited. This lack of coordination may in part be responsible for low rates of uptake of cessation pharmacotherapy benefits. ${ }^{13}$ Better coordination of smoking cessation counseling with the health care team could improve access to pharmacotherapy and resultant smoking cessation.

A meta-analysis of smoking cessation studies has demonstrated the benefit of counseling that begins during the hospital stay and extends at least 4 weeks after a hospital discharge; however, 6 months post-discharge, $70-90 \%$ of smokers will have resumed smoking despite delivery of optimal therapy. ${ }^{3}$ Previous studies suggest that persistent smokers may benefit from re-engagement in treatment, ${ }^{14,}{ }^{15}$ but no studies to date have looked at the impact of re-engaging hospitalized smokers or looked at the impact of counseling and care coordination as a tool for re-engaging smokers.

The purpose of this study was to test the effects of counseling with care coordination (CCC) versus counseling only (C) on pharmacotherapy utilization and smoking cessation outcomes. We also examined the impact of re-engaging persistent smokers 6 months after their hospital discharge.

\section{METHODS}

The study design, including recruitment, counseling, and assessment, procedures have been reported previously. ${ }^{16}$ All study procedures were reviewed and approved by the KUMC institutional review board. The trial was registered at www. clinicaltrials.gov, No. NCT01063972.

\section{Setting and Participants}

We recruited 31, primarily rural, hospitals across the state of Kansas to participate in this study. We worked with a smoking cessation champion at each hospital to develop a plan for identifying and referring smokers. We provided an orientation of the program for the medical staff at each hospital and conducted in-services with nursing personnel. We tracked referrals over time from each hospital and helped staff troubleshoot problems with recruitment. All participants were recruited between April 2010 and October 2013.

We asked staff at participating hospitals to identify all smokers and ask if they would be willing to speak to someone from the University of Kansas Medical Center (KUMC) about a research project for smokers. In order to increase the representativeness of smokers, patients were informed that they would not be required to quit to be in the study, but that the program was designed to help smokers quit. For willing smokers, the hospital staff provided a smoking cessation guide with information about the study and faxed patient contact information to a KUMC research assistant. The research assistant made repeated efforts to contact the patient by telephone while they were still in the hospital and up to 2 business days post-discharge. Upon reaching the potential participant, the research assistant described the study, assessed eligibility, and elicited verbal informed consent based on a consent document included in the smoking cessation guide provided at the time of referral. Smokers were eligible to participate if they were $\geq 18$ years of age, smoked cigarettes on $\geq 25$ of the last 30 days prior to admission, and had a home address and telephone. We excluded smokers who were pregnant, terminally ill, or being discharged to a nursing facility where they could not smoke.

Eligible, consenting participants completed the baseline assessment and were randomly assigned to receive either smoking cessation counseling only (C) or smoking cessation counseling with care coordination (CCC). We randomized patients in blocks of 4 at each hospital to reduce the potential for confounding by treatment site.

\section{Interventions}

All participants received a smoking cessation guide that addressed the benefits of quitting and self-help strategies for quitting and staying quit. In addition, the guide provided information on smoking cessation pharmacotherapy and pharmacy assistance programs that could provide free pharmacotherapy for low-income patients. All participants were offered telephone-based counseling scheduled at enrollment and day 2 and weeks 1, 3, and 6 post-enrollment. All participants were offered an additional counseling session following their month 6 assessment. A second cycle of telephone-based counseling was then offered at 1,3 , and 6 weeks after the month 6 assessment for persistent or relapsed smokers or those who had been quit less than 90 days, in addition to any who were on cessation medications regardless of how long they had been quit. Smoking cessation pharmacotherapy was not provided directly through the study; participants needed to procure pharmacotherapy on their own or through their health care provider. Support for procuring pharmacotherapy varied according to treatment arm as described below.

Ongoing counseling supervision was provided by a clinical psychologist and treatment fidelity was assessed by reviewing session recordings and documentation of care coordination. Supervisors completed fidelity rating forms, which evaluated global tobacco treatment skills, as well as compliance with research protocols by treatment arm for counseling and care coordination.

Counseling (C). Participants randomized to counseling alone (C) received individualized, telephone-based counseling designed to emulate protocols used by existing telephone 
quitlines. Each session focused on enhancing motivation and helping smokers interested in quitting develop a quit plan that addressed environmental changes, social support, pharmacotherapy, cognitive-behavioral coping skills, and relapse prevention strategies. Pharmacotherapy use was advised for those implementing a quit plan and counselors referred interested participants to the relevant pages in the smoking cessation guide, and discussed treatment options. For participants reporting current pharmacotherapy use, adherence and side effect management were discussed. For $\mathrm{C}$ participants, the study counselor did not communicate directly with the hospital or the participant's health care provider, but participants were advised on how they could obtain pharmacotherapies either over the counter or from their health care provider.

Counseling with Care Coordination. The same group of cessation counselors provided cessation counseling to the participants in the counseling with care coordination (CCC) arm. In addition to the smoking cessation counseling described above for the $\mathrm{C}$ participants, the $\mathrm{CCC}$ intervention provided additional care coordination, including screening for contraindications to different types of pharmacotherapy and collecting information on the types of smoking cessation treatments covered by the participant's insurance plan. Based on this information, counselors provided a tailored list of options with a strong recommendation for pharmacotherapy using an "opt out" approach. ${ }^{17}$ With the opt out approach, counselors asked participants which type of pharmacotherapy they would like to use from the list, rather than if they would like to use pharmacotherapy. After helping the participant choose his or her desired treatment, the counselor faxed a prescription request to the participant's health care provider along with a patient action plan summarizing the counseling session. Baseline inpatient requests were sent to attending physicians and post-discharge requests were sent to the patient's primary care physician. Additional prescription requests and patient action plans were sent to the provider following subsequent counseling calls to prompt medication refills or if a medication change was warranted. Counselors sent a counseling report to the participant's physician twice during each cycle of counseling calls.

\section{Measures}

Participants completed assessments via telephone at baseline and at 3, 6, and 12 months post-discharge. The baseline assessment included information on demographics, smoking characteristics, hospitalization characteristics, and health history (Table 1). At 3, 6, and 12 months post-discharge, participants were asked about any interim use of smoking cessation pharmacotherapy. Participants were asked separately about their use of varenicline, bupropion, nicotine patch, or short-acting nicotine replacement therapy (NRT) (i.e., nicotine gum, lozenge, nasal spray, or inhaler), or use of more than one agent. Participants were asked about self-reported, 7-day point prevalence abstinence at 3,6, and 12 months. Participants reporting abstinence at 12 months were asked to mail in a saliva sample for measurement of salivary cotinine. A salivary cotinine level of $<15 \mathrm{ng} / \mathrm{ml}$ was used to confirm abstinence. For participants unable or unwilling to provide salivary cotinine, we contacted a previously designated proxy to confirm their smoking status.

\section{Data Analysis}

All analyses were completed using SAS version 9.4, SAS Institute Inc., Cary, NC, USA.

We compared utilization of pharmacotherapy and selfreported abstinence across treatment groups at 3, 6, and 12 months using chi-square. The primary, a priori outcome for this study was validated (by salivary cotinine or proxy), 7day point prevalence abstinence at 12 months. For cessation analyses, we excluded participants who had died or were incarcerated at that time point. Other non-respondents were imputed to be smokers. Assessments of pharmacotherapy utilization were restricted to respondents only. We used an alpha level of .05 for all statistical tests.

The sample size was based on the primary outcome with estimated quit rates of $12 \%$ and $21 \%$ in the $\mathrm{C}$ and $\mathrm{CCC}$ arms, respectively. With a two-tailed, type 1 error set at 0.05 , a sample size of 576 participants ( 288 per arm) would provide a power of $80 \%$ to detect a difference of this magnitude or greater. We anticipated excluding patients who expired prior to follow-up and consequently oversampled by $5 \%$, leading to our final sample size of 606 participants.

\section{RESULTS}

Of the 1652 smokers referred to the program, 345 (20.9\%) declined screening. Of the 1307 screened, 254 (19.4\%) declined enrollment, 447 (34.2\%) were ineligible, and 606 $(46.4 \%)$ were randomized to either CCC $(n=310)$ or counseling alone $(n=296)$ (Fig. 1). The two treatment arms were similar at baseline (Table 1). On average, participants were $50.8(\mathrm{SD}=12.37)$ years old; the majority were female $(65 \%)$ and White $(90 \%)$. The majority $(55 \%)$ had a high school education or less, $41 \%$ lived below the federal poverty level, and $76 \%$ had health insurance. At baseline, participants smoked an average of 19.4 cigarettes per day $(\mathrm{SD}=10.41)$, and $89 \%$ indicated their intention of using this hospitalization as an opportunity to quit. Follow-up at all time points was comparable between the two treatment groups (Fig. 1) with $499(82.3 \%), 466(76.9 \%)$, and $429(70.8 \%)$ completing follow-up at 3, 6, and 12 months, respectively. After excluding patients who died or were incarcerated, 580 (95.7\%) were included in the primary analysis. 
Table 1 Baseline Characteristics of Study Participants

\begin{tabular}{|c|c|c|c|}
\hline & \multirow{2}{*}{$\begin{array}{l}\begin{array}{l}\text { Total } \\
n=606\end{array} \\
\text { No. }(\%)\end{array}$} & \multirow{2}{*}{$\begin{array}{l}\begin{array}{l}\text { Counseling } \\
n=296\end{array} \\
\text { No. }(\%)\end{array}$} & \multirow{2}{*}{$\begin{array}{l}\begin{array}{l}\text { CDM } \\
n=310\end{array} \\
\text { No. }(\%)\end{array}$} \\
\hline & & & \\
\hline \multicolumn{4}{|l|}{ Patient characteristics } \\
\hline Age, years; mean (SD) & $50.8(12.37)$ & $51.0(11.82)$ & $50.6(12.89)$ \\
\hline Gender, female & $393(65)$ & $189(64)$ & $194(63)$ \\
\hline \multicolumn{4}{|l|}{ Race } \\
\hline White & $548(90)$ & $270(91)$ & $278(90)$ \\
\hline African-American & $26(4)$ & $12(4)$ & $14(5)$ \\
\hline American Indian & $24(4)$ & $12(4)$ & $12(4)$ \\
\hline Other/not reported & $8(1)$ & $2(1)$ & $6(2)$ \\
\hline Latino/a & $34(6)$ & $9(3)$ & $25(8)$ \\
\hline \multicolumn{4}{|l|}{ Employment } \\
\hline Employed & $249(41)$ & $124(42)$ & $125(40)$ \\
\hline Other ${ }^{\mathrm{a}}$ & $357(59)$ & $172(58)$ & $185(60)$ \\
\hline$\leq$ High school graduate & $331(55)$ & $150(51)$ & $181(58)$ \\
\hline Less than $100 \%$ of Federal poverty level & $250(41)$ & $117(40)$ & $133(43)$ \\
\hline Less than $200 \%$ of Federal poverty level & $433(71)$ & $206(70)$ & $227(73)$ \\
\hline Have health insurance & $461(76)$ & $230(78)$ & $231(75)$ \\
\hline Medicaid & $164(27)$ & $82(28)$ & $82(26)$ \\
\hline Have prescription insurance & $428(71)$ & $216(73)$ & $212(68)$ \\
\hline \multicolumn{4}{|l|}{ Marital status } \\
\hline Married/partner & $315(52)$ & $163(55)$ & $152(49)$ \\
\hline Divorce/separated & $196(32)$ & $91(31)$ & $105(34)$ \\
\hline Widowed & $50(8)$ & $24(8)$ & $26(8)$ \\
\hline Never been married & $45(7)$ & $18(6)$ & $27(9)$ \\
\hline \multicolumn{4}{|l|}{ Living status ${ }^{\mathrm{b}}$} \\
\hline Lives alone & $133(22)$ & $57(19)$ & $76(25)$ \\
\hline Other smokers in household & $274(45)$ & $141(48)$ & $133(43)$ \\
\hline Only non-smokers in the home & $198(33)$ & $97(33)$ & $101(33)$ \\
\hline Children $<$ age 12 in home & $142(23)$ & $66(22)$ & $76(25)$ \\
\hline Smoking prohibited in home & $214(35)$ & $109(37)$ & $105(34)$ \\
\hline \multicolumn{4}{|l|}{ Smoking characteristics } \\
\hline Cigarettes smoked per day, mean (SD) & $19.4(10.41)$ & $19.7(10.84)$ & $19.3(9.99)$ \\
\hline Smoke within $30 \mathrm{~min}$ of waking & $501(83)$ & $244(82)$ & $257(83)$ \\
\hline Fagerström score, heavy dependence ${ }^{\mathrm{c}}$ & $280(46)$ & $168(57)$ & $142(46)$ \\
\hline Motivation to quit, mean (SD) ${ }^{\mathrm{d}}$ & $9.3(1.47)$ & $9.2(1.60)$ & $9.3(1.34)$ \\
\hline Confidence to quit, mean (SD) ${ }^{\mathrm{d}}$ & $7.0(2.53)$ & $6.8(2.45)$ & $7.1(2.61)$ \\
\hline Planning to quit in the next 30 days & $588(97)$ & $285(96)$ & $303(98)$ \\
\hline Quit attempted in the past 6 months & $323(53)$ & $154(52)$ & $169(55)$ \\
\hline \multicolumn{4}{|l|}{ Previous use of cessation pharmacotherapy } \\
\hline Any pharmacotherapy & $391(65)$ & $199(67)$ & $192(62)$ \\
\hline Nicotine replacement therapy & $365(60)$ & $175(59)$ & $190(61)$ \\
\hline Bupropion & $150(25)$ & $85(29)$ & $65(21)$ \\
\hline Varenicline & $218(36)$ & $117(40)$ & $101(33)$ \\
\hline \multicolumn{4}{|l|}{ Patient's doctor... } \\
\hline Understands my feelings about smoking ${ }^{\mathrm{e}}$ & $366(60)$ & $182(62)$ & $184(59)$ \\
\hline Thinks it's important for me to quit ${ }^{\mathrm{e}}$ & $509(84)$ & $254(86)$ & $255(82)$ \\
\hline Takes time to talk about smoking ${ }^{\mathrm{e}}$ & $372(61)$ & $189(64)$ & $183(59)$ \\
\hline \multicolumn{4}{|l|}{ Hospitalization characteristics } \\
\hline Length of hospital stay (days), mean (SD) & $4.5(6.14)$ & $4.3(4.25)$ & $4.6(7.52)$ \\
\hline Planning to use hospitalization as an opportunity to quit & $541(89)$ & $260(88)$ & $281(91)$ \\
\hline Relates current hospitalization to smoking ${ }^{\mathrm{e}}$ & $200(33)$ & $99(33)$ & $101(33)$ \\
\hline Believes quitting smoking will help with their recovery ${ }^{\mathrm{e}}$ & $463(76)$ & $228(77)$ & $235(76)$ \\
\hline Difficult to not smoke while in the hospital & $134(22)$ & $58(20)$ & $76(25)$ \\
\hline Moderate/severe cravings while in the hospital & $215(35)$ & $104(35)$ & $111(36)$ \\
\hline Receipt of smoking medications during hospitalization & $213(35)$ & $102(34)$ & $111(36)$ \\
\hline \multicolumn{4}{|l|}{ Health history } \\
\hline Rate current health as "fair" or "poor" & $348(57)$ & $170(57)$ & $178(57)$ \\
\hline Diabetes & $162(27)$ & $84(28)$ & $78(25)$ \\
\hline Chronic lung disease & $301(50)$ & $135(46)$ & $166(54)$ \\
\hline Heart disease & $148(24)$ & $69(23)$ & $79(25)$ \\
\hline History of seizure & $82(14)$ & $41(14)$ & $41(13)$ \\
\hline History of depression & $309(51)$ & $155(52)$ & $154(50)$ \\
\hline Current depression (PHQ-2 score $\geq 3)^{\mathrm{f}}$ & $262(43)$ & $121(41)$ & $141(45)$ \\
\hline FAST $>3$, & $59(10)$ & $27(9)$ & $32(10)$ \\
\hline
\end{tabular}

${ }^{a}$ Other includes currently not employed, homemaker, student, retired, and disabled

${ }^{b}$ Living status is unknown for 1 participant in the counseling group

${ }^{c}$ The modified Fagerström test score for nicotine dependence ranges from 0 to 6. Scores of 4 or higher indicate heavy smoking dependence ${ }^{18}$

${ }^{d}$ Motivation and confidence to quit smoking scores range from 0 to 10

${ }^{e}$ Rate agreement based on a response of 6 or 7 on a 7-point Likert scale

${ }^{f}$ Patient Health Questionnaire-2 (PHQ-2) Depression Screening Tool score $\geq 3$ indicates possible diagnosis of depression ${ }^{19}$

${ }^{g}$ Fast Alcohol Screening Test score of 3 or more indicates alcohol use disorder ${ }^{20}$ 


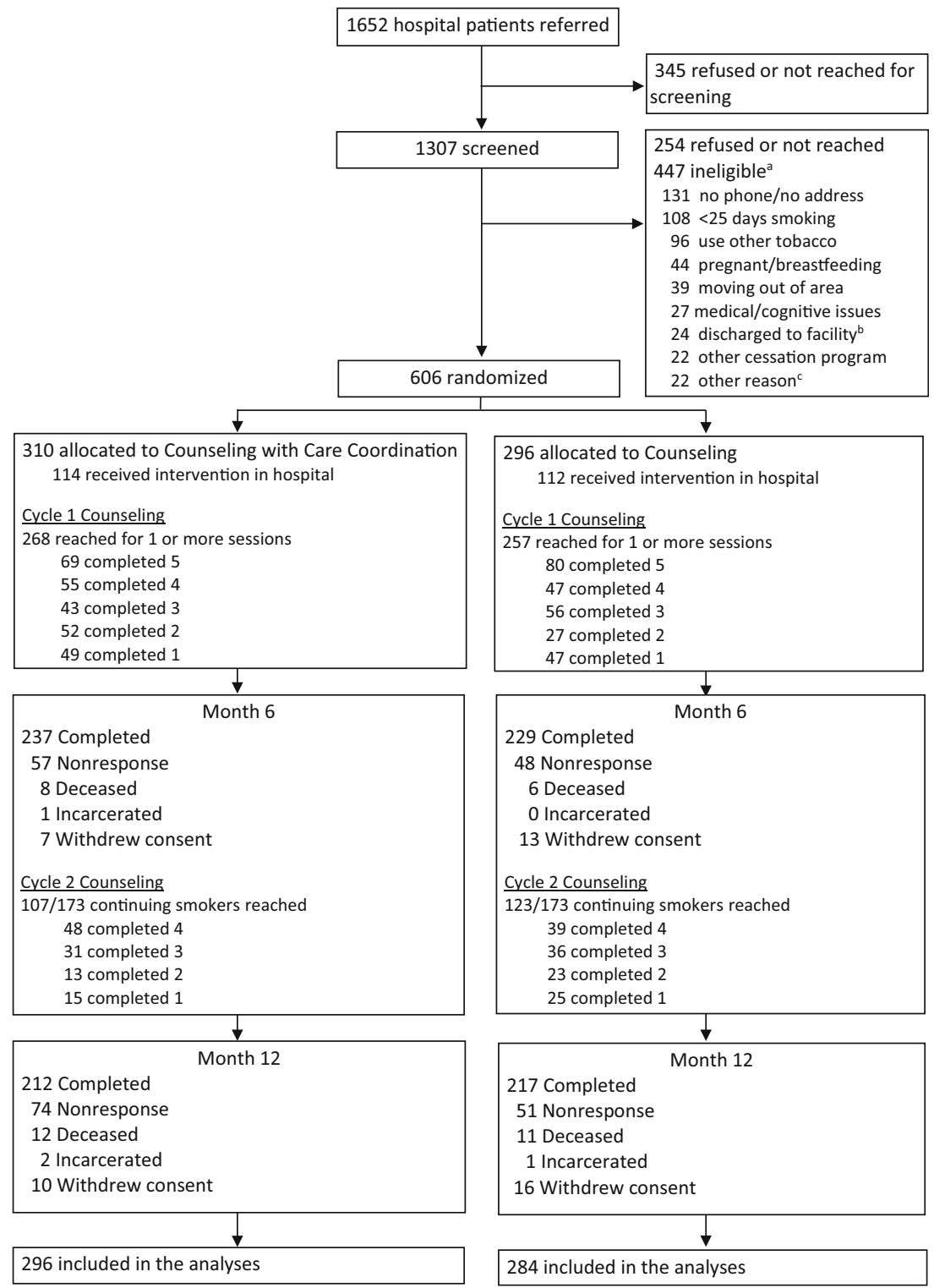

Figure 1 CONSORT diagram. Deceased, incarcerated, and withdrew consent are reported cumulatively. Deceased and incarcerated were removed from analyses. ${ }^{a}$ Ineligible categories are not mutually exclusive. ${ }^{\mathrm{b}}$ Discharge facilities were primarily nursing homes, but also included incarceration and alcohol or drug rehabilitation. " “Other" includes prolonged hospital stay, under 18 years of age, emergency room visit only, and already enrolled.

\section{Uptake of Counseling}

Participants completed an average of $2.81(\mathrm{SD}=1.76)$ cessation counseling calls during the first 3 months after enrollment with similar uptake of counseling calls in the CCC $(M=2.73$, $\mathrm{SD}=1.74)$ and $\mathrm{C}(M=2.90, \mathrm{SD}=1.78)$ groups $(p=0.25)$. Despite attempts to enroll and counsel patients immediately upon referral, 380 (62.7\%) did not receive their first counseling session during their hospitalization, including 196 (63.2\%) of those in the CCC group who consequently were not able to receive any care coordination until after discharge. The 346 participants who reported smoking at 6 months completed an average of 1.91 (SD 1.63) additional counseling calls with no difference in the uptake of counseling across the two treatment arms $(p=0.77)$ (Fig. 1).

\section{Uptake of Pharmacotherapy}

Use of any cessation pharmacotherapy between 0 and 6 months $(55.2 \%)$ and between 6 and 12 months $(47.1 \%)$ post-discharge was similar across treatment arms. During the first 6 months after discharge, $54.9 \%$ of participants in the CCC group and $55.5 \%$ of those in the C group reported using pharmacotherapy for smoking cessation (RR, 0.98 (95\% CI, 0.85, 1.16)) (Table 2). Of 314 continuing smokers at month 6 that completed the month 12 assessment, $68(42.2 \%)$ of the C participants and 80 $(52.3 \%)$ of the CCC participants reported use of pharmacotherapy during the final 6 months of follow-up (i.e., after the second cycle of counseling) (RR, 1.23 (95\% CI, $0.98,1.57)$ ). Most participants utilized over-the-counter 
Table 2 Number (\%) of Smokers Reporting Use of Pharmacotherapy During the 6 Months After Hospitalization and, for Continuing Smokers, 6-12 Months After Discharge

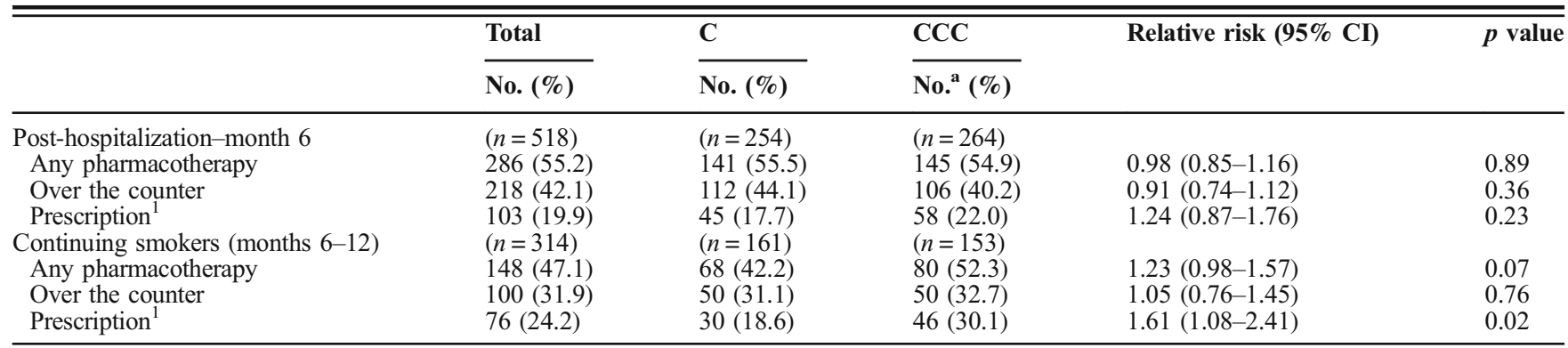

${ }^{1}$ Varenicline or bupropion

$C$, counseling only; CCC, counseling plus care coordination

cessation products with no statistically significant difference between treatment arms. Between months 6 and 12, however, significantly more CCC participants reported use of prescription-only pharmacotherapy $(30.1 \%$ vs. $18.6 \%$; RR 1.61, 95\% CI, 1.08, 2.41)).

\section{Smoking Cessation}

Participants reported as deceased or incarcerated $(n=26)$ were excluded from analyses, leaving 580 in the final analyses. Comparable numbers of participants in the CCC arm and the $\mathrm{C}$ arm achieved the primary outcome of validated smoking cessation at the 12-month followup $(19.3 \%$ vs. $16.9 \%$, respectively, RR, 1.13 (95\% CI, $0.80,1.61)$ ). Self-reported abstinence rates were comparable across treatment arms at 3, 6, and 12 months (Table 3). Overall, self-reported abstinence declined from $26.2 \%$ at month 3 to $20.3 \%$ at month 6 and then increased to $23.4 \%$ at month 12 . Changes in selfreported smoking status from month 6 to month 12 are shown in Table 4. Of the 118 smokers self-reporting abstinence at month $6,17(14.4 \%)$ reported a relapse to smoking at month 12 ; of the 337 participants that were smoking at month 6 and survived to month 12,42 $(12.5 \%)$ reported that they were abstinent at month 12 , i.e., new quitters.

\section{DISCUSSION}

Telephone-delivered care management, provided by a counselor outside of the immediate health care team, did not outperform standard telephone-delivered counseling for smoking cessation among hospitalized smokers. Supplemental care coordination did not increase overall use of pharmacotherapy over standard counseling either during the hospitalization or after discharge, but was associated with higher use of prescription-only pharmacotherapy among continued smokers that were re-engaged in treatment 6 months post-discharge.

There are a number of possible reasons that care management may have failed to outperform standard counseling in this study. First, participants were highly motivated to quit. In this context, the encouragement offered by standard counseling to use medications may have been powerful enough to achieve moderately high rates of medication use in the control arm, in effect overwhelming the additional assistance offered by care management.

Second, due to short hospital stays and rapid discharges, most participants failed to receive counseling in the hospital where care management might have been most likely impact medication utilization. ${ }^{16}$ After discharge, care managers were unable to coordinate with hospital staff to procure prescriptions. Patients who use cessation medications in the hospital are more likely to use them post-discharge. ${ }^{16}$ This missed opportunity to coordinate care may have contributed to

Table 3 Self-reported and Validated Abstinence Rates

\begin{tabular}{|c|c|c|c|c|c|}
\hline & Total & $\mathbf{C}$ & $\mathrm{CCC}$ & \multirow[t]{2}{*}{ Relative risk (95\% CI) } & \multirow[t]{2}{*}{$p$ value } \\
\hline & No. ${ }^{a}(\%)$ & No. ${ }^{a}(\%)$ & No. ${ }^{a}(\%)$ & & \\
\hline \multicolumn{6}{|c|}{ Self-reported 7-day point prevalence } \\
\hline Month 3 & $156 / 595(26.2)$ & $70 / 292(24.0)$ & $86 / 303(28.4)$ & $1.18(0.90-1.55)$ & 0.22 \\
\hline Month 6 & $120 / 591(20.3)$ & $56 / 290(19.3)$ & $64 / 301(21.3)$ & $1.10(0.80-1.52)$ & 0.56 \\
\hline Month 12 & $136 / 580(23.4)$ & $63 / 284(22.2)$ & $73 / 296(24.7)$ & $1.11(0.83-1.49)$ & 0.48 \\
\hline \multicolumn{6}{|c|}{ Validated 7-day point prevalence ${ }^{\mathrm{b}}$} \\
\hline Month 12 & $105 / 580(18.1)$ & 48/284 (16.9) & $57 / 296(19.3)$ & $1.13(0.80-1.61)$ & 0.46 \\
\hline
\end{tabular}

$C$, counseling only; $C C C$, counseling plus care coordination

Denominators for self-reported prevalence include participants who completed the survey and were lost to follow-up. Numerator includes participants who reported abstinence

${ }^{a}$ Excludes deceased or incarcerated at each time point. See Figure 1

${ }^{b}$ Biochemically validated by salivary cotinine $(<15 \mathrm{ng} / \mathrm{ml})$ or proxy 
Table 4 Changes in Self-reported Smoking Status Between Month 6 and Month 12

\begin{tabular}{|c|c|c|c|c|}
\hline \multirow[t]{3}{*}{ Status at month 6} & \multirow[t]{3}{*}{$n=580^{a}$} & \multicolumn{3}{|c|}{ Status at month 12} \\
\hline & & \multirow{2}{*}{$\begin{array}{l}\begin{array}{l}\text { Smoking } \\
n=293\end{array} \\
\text { No. }(\%)\end{array}$} & \multirow{2}{*}{$\begin{array}{l}\text { Abstinent } \\
n=136 \\
\text { No. }(\%)\end{array}$} & \multirow{2}{*}{$\begin{array}{l}\text { Nonresponse } \\
n=151 \\
\text { No. }(\%)\end{array}$} \\
\hline & & & & \\
\hline Smoking & 337 & $247(73.3)$ & $42(12.5)$ & 48 (14.2) \\
\hline Abstinent & 118 & $17(14.4)$ & $90(76.3)$ & $11(9.3)$ \\
\hline Nonresponse & 125 & $29(23.2)$ & $4(3.2)$ & $92(73.6)$ \\
\hline
\end{tabular}

${ }^{a}$ Excludes deceased or incarcerated $(n=26)$

equivalent relapse rates post-hospitalization among the care management and counseling-only arms.

Third, patients who were issued prescription requests may never have had them filled. This study was designed as a pragmatic study taking advantage of existing sources of pharmacotherapy, and participants were either dependent upon their insurance to pay for their pharmacotherapy or they needed to pay for their treatment out of pocket. Both patients and providers are confused about smoking cessation insurance benefits ${ }^{8,11}$ and uptake of smoking cessation benefits has been very low, with only about $3 \%$ of Medicaid smokers in Kansas using any type of cessation therapy in $2013 .{ }^{13}$ Our counseling-only arm was designed to mimic standard telephone quitline protocols and strongly recommended that smokers use pharmacotherapy for a quit attempt, but they did not provide pharmacotherapy directly or provide assistance in procuring treatment.

At the time of enrollment, $89 \%$ of the smokers indicated a desire to use the hospitalization to quit, but only $35 \%$ took advantage of pharmacotherapy during the hospitalization and $55.1 \%$ after discharge. These rates of pharmacotherapy uptake are much lower than that seen in settings where pharmacotherapy was provided directly and without cost. ${ }^{21}$ Our supplemental care coordination focused exclusively on the patient and their medical provider (i.e., getting a prescription written for their smoking cessation medication). We did not address residual barriers in actually getting the prescription filled at the pharmacy. Our care coordinators looked at insurance coverage by type of medication (e.g., varenicline, bupropion, or nicotine patch) but did not look at coverage of specific brands or formulations (e.g., covered NDC codes). ${ }^{9}$ Even though a smoking cessation product is covered by their insurance, a smoker may still encounter difficulties in getting a prescription filled at the pharmacy. ${ }^{11,22}$ Coverage, particularly for NRT, varies by brand and packaging, and it can be difficult for pharmacists to identify which particular product options, if any, are actually covered by any given patient's insurance plan. ${ }^{23}$ It is possible that additional care coordination to address barriers at the pharmacy might have resulted in different outcomes.

Consistent with other smoking cessation studies, ${ }^{15,} 21$ abstinence rates in our study decreased between months 3 and 6 post-discharge. A unique aspect of our study was the effort to re-engage persistent or relapsed smokers in treatment 6 months post-discharge. Our study demonstrated strong interest among smokers in this re-engagement with almost half of the persistent smokers using pharmacotherapy and engaging in at least 3 additional counseling calls. Although we did not see a difference by treatment arm, these modest efforts were associated with $12.5 \%$ of persistent smokers at month 6 reporting they were abstinent at month 12 . These findings are consistent with other studies showing the benefits of ongoing re-engagement of persistent smokers. ${ }^{14,15,24}$

\section{Limitations}

Our study has a number of limitations. We were dependent upon referral of patients and likely did not receive a representative sample of the smokers seen at our participating hospitals. Patients referred to us may have been more motivated to quit and already receiving smoking cessation therapy. Our care coordinators were not members of the immediate treatment team and findings might be different for care coordinators embedded within the same health system. Counseling in both study arms commenced for most participants post-discharge and limited our ability in many cases to take advantage of medication coordination the hospitalization itself. The absence of a centralized electronic health record precluded us from capturing changes in processes of care related to $\mathrm{CCC}$ such as changes in office visits or prescriptions. Implementation of the Affordable Care Act 25,26 and increased use of e-cigarettes since the completion of this study might also affect the findings.

\section{CONCLUSION}

Despite a strong interest and motivation to quit, many smokers in this study did not receive pharmacotherapy after hospital discharge. Supplemental, phone-based care coordination failed to address this problem and did not improve overall pharmacotherapy utilization or smoking cessation beyond that achieved by more traditional, telephone-delivered cessation counseling. Increases in abstinence rates when persistent smokers are re-engaged in treatment 6 months postdischarge suggest the need to follow hospitalized smokers long-term and re-engage persistent smokers in treatment. During this re-engagement, supplemental care coordination may increase uptake of prescription pharmacotherapy. 
Acknowledgments: The authors would like to thank the hospitals throughout the state of Kansas that participated in this study and the volunteers who participated in this research.

Corresponding Author: Edward F. Ellerbeck, MD, MPH; Department of Population Health University of Kansas Medical Center, 3901 Rainbow Blvd., MS 1008, Kansas City, KS 66160, USA (e-mail: eellerbe@kumc.edu).

Funding This work was supported by the National Cancer Institute of the National Institutes of Health grant number R01CA101963. The content is solely the responsibility of the authors and does not necessarily represent the official view of the National Institutes of Health. This trial was registered on clinicaltrials.gov (NCTO1063972).

\section{Compliance with Ethical Standards:}

All study procedures were reviewed and approved by the KUMC institutional review board.

Conflict of Interest: Drs. Ellerbeck, Cox, Hui, Keighley, Hutcheson, Cupertiono, Greiner, Miller, Rabius, and Richter and Ms. Fitzgerald report grants from the National Institutes of Health during the conduct of the study.

Dr. Rigotti reports grants from NCI, during the conduct of the study; personal fees from UpToDate, Inc.; personal fees from Achieve Life Sciences; and grants and non-financial support from Pfizer, outside the submitted work.

\section{REFERENCES}

1. Benowitz NL. Cigarette smoking and cardiovascular disease: pathophysiology and implications for treatment. Prog Cardiovasc Dis. 2003;46(1):91-111

2. Hennrikus DJ, Lando HA, McCarty MC, et al. The TEAM project: the effectiveness of smoking cessation intervention with hospital patients. Prev Med. 2005;40(3):249-258.

3. Rigotti NA, Munafo MR, Stead LF. Smoking cessation interventions for hospitalized smokers: a systematic review. Arch Intern Med. 2008;168(18):1950-1960.

4. Rigotti NA, Clair C, Munafo MR, Stead LF. Interventions for smoking cessation in hospitalised patients. Cochrane Database Syst Rev. 2012;5:CD001837.

5. Fiore MC, Jaén CR, Baker TB, et al. Treating tobacco use and dependence: 2008 update U.S. Public Health Service Clinical Practice Guideline executive summary. Respir Care. 2008;53(9):1217-1222.

6. Snow V, Beck D, Budnitz T, et al. Transitions of Care Consensus policy statement: American College of Physicians, Society of General Interna Medicine, Society of Hospital Medicine, American Geriatrics Society, American College Of Emergency Physicians, and Society for Academic Emergency Medicine. J Hosp Med. 2009;4(6):364-370.

7. DiGiulio A, Jump Z, Yu A, et al. State Medicaid Coverage for Tobacco Cessation Treatments and Barriers to Accessing Treatments - United States, 2015-2017. MMWR Morb Mortal Wkly Rep. 2018;67(13):390395.

8. McMenamin SB, Halpin HA, Ibrahim JK, Orleans CT. Physician and enrollee knowledge of Medicaid coverage for tobacco dependence treatments. Am J Prev Med. 2004;26(2):99-104.
9. Murphy JM, Mahoney MC, Hyland AJ, Higbee C, Cummings KM. Disparity in the use of smoking cessation pharmacotherapy among Medicaid and general population smokers. J Public Health Manag Pract. 2005;11(4):341-345.

10. North American Quitline Consortium 2015. (Accessed October 13, 2007, at http://www.naquitline.org/). Accessed 1 June 2015

11. Sheffer CE, Anders M, Brackman SL, Steinberg MB, Barone C. Tobacco intervention practices of primary care physicians treating lower socioeconomic status patients. Am J Med Sci. 2012;343(5):388-396.

12. Miller N, Frieden TR, Liu SY, et al. Effectiveness of a large-scale distribution programme of free nicotine patches: a prospective evaluation. Lancet. 2005;365(9474): 1849-1854.

13. Ku L, Bruen BK, Steinmetz E, Bysshe T. Medicaid Tobacco Cessation: Big Gaps Remain In Efforts To Get Smokers To Quit. Health Aff (Millwood). 2016;35(1):62-70.

14. Ellerbeck EF, Mahnken JD, Cupertino AP, et al. Effect of varying levels of disease management on smoking cessation: a randomized trial. Ann Intern Med. 2009;150(7):437-446.

15. Joseph AM, Fu SS, Lindgren B, et al. Chronic disease management for tobacco dependence: a randomized, controlled trial. Arch Intern Med. 2011;171(21): 1894-1900.

16. Tague C, Richter KP, Cox LS, et al. Impact of Telephone-Based Care Coordination on Use of Cessation Medications Posthospital Discharge: A Randomized Controlled Trial. Nicotine Tob Res. 2016.

17. Richter KP, Ellerbeck EF. It's time to change the default for tobacco treatment. Addiction. 2015;110(3):381-386.

18. Heatherton TF, Kozlowski LT, Frecker RC, Fagerstrom KO. The Fagerstrom Test for Nicotine Dependence: a revision of the Fagerstrom Tolerance Questionnaire. Br J Addict. 1991;86(9):1119-1127.

19. Kroenke K, Spitzer RL, Williams JB. The Patient Health Questionnaire2: validity of a two-item depression screener. Med Care. 2003;41(11):1284-1292.

20. Hodgson R, Alwyn T, John B, Thom B, Smith A. The FAST Alcohol Screening Test. Alcohol Alcohol. 2002;37(1):61-66.

21. Rigotti NA, Regan S, Levy DE, et al. Sustained care intervention and postdischarge smoking cessation among hospitalized adults: a randomized clinical trial. Jama. 2014;312(7):719-728.

22. Singleterry J, Jump Z, DiGiulio A, et al. State Medicaid Coverage for Tobacco Cessation Treatments and Barriers to Coverage - United States, 2014-2015. MMWR Morb Mortal Wkly Rep. 2015;64(42):1194-1199.

23. Luthra S. Lack of Medical Expansion Hurts Rural Hospitals More than Urban Facilities. Kaiser Health News. 2016;35(1):62-70.

24. Cupertino AP, Wick JA, Richter KP, Mussulman L, Nazir N, Ellerbeck EF. The impact of repeated cycles of pharmacotherapy on smoking cessation: a longitudinal cohort study. Arch Intern Med. 2009; 169(20):1928-1930.

25. Lemaire RH, Bailey L, Leischow SJ. Meeting the Tobacco Cessation Coverage Requirement of the Patient Protection and Affordable Care Act: State Smoking Cessation Quitlines and Cost Sharing. Am J Public Health. 2015;105 Suppl 5:S699-705.

26. Kofman M DK, Senkewicz MB. Implementation of Tobacco Cessation Coverage Under the Affordable Care Act: Understanding How Private Health Insurance Policies Cover Tobacco Cessation Treatment. Washington, DC: Georgetown University Health Policy Institute; 2012.

Publisher's Note Springer Nature remains neutral with regard to jurisdictional claims in published maps and institutional affiliations. 